\title{
Effects of Essential Oil Extracted from Herb on Job Stress, Depression and Fatigue of Hospital Workers
}

\author{
Kyung-Ja Kim', Suk-Young Yun ${ }^{2}$ *, Byung-Jin Choi ${ }^{2}$, and Jeong-Eon Park ${ }^{3}$ \\ ${ }^{1}$ Kyungpook National University Hospital, Daegu 41944, South Korea \\ ${ }^{2}$ Faculty of Environment, Horticulture and Landscaping, Catholic University of Daegu, Kyungsan 38430, South Korea \\ ${ }^{3}$ Department of Nursing, Uiduk University, Gyeongju 38004, South Korea
}

\begin{abstract}
This study was implemented to verify effectiveness related to job stress, depression or fatigue of hospital workers, by let them wearing necklaces with essential oils injected which were extracted from herb plants, as an alternative therapy. For this study, totally 60 people were selected who work in $\mathrm{K}$ hospital located in D city, in accordance with their agreement with participation in, and who were divided into 30 and 30 for a control group and an experimental group as appropriate subjects. The study was followed for 2 weeks from June 29 of 2015 by July 10 of 2015, by letting the experimental group wearing essential oils necklaces with a distance of $20 \mathrm{~cm}$ from noses during working time for 8 hours per day. After inhaling essential oils by wearing the necklaces, the job stress status didn't have obvious difference between two groups statically, such as $104.1 \pm 11.3$ points from $101.2 \pm 10.3$ points for the control group and $104.7 \pm 9.8$ points from $104.9 \pm 9.0$ points for the experimental group. In case of the depression status, the control group had no difference such as $28.8 \pm 8.0$ points from $29.4 \pm 9.0$ points, and the experimental group had small decrease with 0.017 points such as $26.6 \pm 5.3$ points from $28.5 \pm 5.6$ points. In case of the fatigue status, the control group had no difference such as $75.7 \pm 19.7$ points from $72.0 \pm 19.0$ points, and the experimental group had small decrease with 0.049 points such as $64.3 \pm 20.0$ points from $70.5 \pm 19.0$ points.
\end{abstract}

Keywords: alternative, herb plants, necklaces, therapy

\section{Introduction}

According to a social survey, $66.6 \%$ of the entire population in Korea is suffering from stress, and $72.9 \%$ of stress is caused by their life at work (Statistics Korea, 2014). Stress can be encountered by anyone, and for workers that spend a great part of the day at work, workplace serves as a key factor of stress in addition to a means of self-achievement and life. The right amount of stress creates certain tension and motivates individuals to grow and increase productivity, but too much stress may not only damage an individual's physical and mental health, but also cause neglect of duty, faults and absence from work. Furthermore, it causes various mental and behavioral responses, and if the level is severe or exceeds the point where the individual can adjust, it may result in mental illness and physical diseases. Severe stress also mentally affects individuals and cause symptoms of depression, and threatens mental health so much that it may even lead to suicide (Yu, 2010). 
A hospital deals with life, death and the process of dying, which exposes its workers to a great deal of job stress. Due to the increase of the government's various regulation policies, intensified competition among medical institutions, changes in national consciousness and IT development, hospitals are becoming more enlarged and specialized. The increasing average lifespan changes the medical trend to prevention of disease, and the increasing cosmetic treatments and diversity of needs from contact with guardians, customer satisfaction and complaints are all increasing job stress for medical personnel or paramedics such as doctors, nurses, medical technicians, and nurse aides. In other words, health workers are experiencing much more job stress than others (Kim and Suh, 2011). Recently, with the improvement of service quality and higher demand for patient satisfaction, efforts are required to regulate and control emotional expressions when providing service for patients, and this emotional pain is considered a certain labor that has also began to be perceived important. Such job stress of hospital workers is accompanied by depression and fatigue, which ultimately leads to lower quality of life, increase in turnover rates, and deteriorated quality of medical services.

Therefore, to apply aroma therapy that is known as an alternative therapy for hospital workers, this study had hospital workers wear a necklace injected with essential oil extracted from herbs, and examined the effects of its aroma on their job stress, depression and fatigue.

\section{Research Method}

\section{Experimental design}

This study adopted the nonequivalent control-group pretest-posttest design to test the effect of inhaling the aroma by wearing necklaces with essential oil extracted from herbs on work stress, depression and fatigue of hospital workers, Both the control group and experimental group had their general characteristics surveyed in advance, after which their job stress, depression and fatigue were measured. The control group did not undergo any treatment, while the experimental group was to inhale the aroma by wearing necklaces with essential oil extracted from herbs. After the treatment, both groups had their job stress, depression and fatigue measured at the same point of time in the same method as before.

\section{Subjects}

To determine the effect of essential oil extracted from herbs on job stress, depression and fatigue of hospital workers, this study selected total 60 subjects -30 each for the control group and experimental group - as the final subjects. The subjects are ordinary workers at $\mathrm{K}$ University Hospital in D city that agreed to participate in this study, and who have no hypersensitive or allergic reaction to aromas, have nothing wrong with olfactory sense, do not take drugs like antidepressants, and are not receiving other treatment such as exercise or yoga.

\section{Research tool}

\section{Inhaling tool}

Aroma necklaces were used as the tool to inhale the aroma of essential oil. The necklaces are easy to carry around and can be inhaled immediately in cases of stress by carrying them around at work (Oh, 2012). For convenience, the necklaces have adjustable straps, and the necklaces and pendants had five options in colors similar to their working clothes to avoid distraction at work. To consistently inhale the aroma of essential oil during the experiment, the length of the necklace was adjusted to keep a $20 \mathrm{~cm}$ distance between the nose and the pendant, with reference to a previous study on the effect of aroma therapy using necklaces on the changes in stress and autonomic nervous system of nursing students in clinical training (Kim et al., 2014), and a study on the effect of aroma pads on stress, fatigue and depression of nurses in operating rooms (Lee, 2012). 


\section{Essential oil}

The essential oils extracted from herbs used as ingredients are top note bergamot (Citrus bergamia (origin; Italy) 100\%, English Kerfoot), middle note lavender (Lavendula angustifolia (origin; Bulgaria) 100\%, Helga Stolz Gmbh), and base note sandalwood (Santalum album (origin; India) 100\%, Australian Kerfoot). The main ingredients of bergamot are esters (Linalyl acetate) and monoterpenes (Limonene) that lift the mood, and thus it is one of the oils that are most preferentially used to relieve depression. The main ingredients of lavender are esters (Linalyl acetate) and alcohols (Linalol) for soothing and relaxing, which are effective for stress, anxiety, insomnia and headaches suffered by people today. The main ingredient of sandalwood is alcohols (Cis- $\alpha$-santalol, Cis- $\beta$-santalol) with almost no toxicity or irritation, and that give active vitalization (Sakong and Kim, 2006). To avoid phased release according to volatility, bergamot, lavender and sandalwood are blended in the ratio of 3:2:1 and matured for 1 week, after which the oils were injected into the aroma necklaces for the subjects in the experimental group to wear. Table 1 shows the main ingredients and blending ratios of the oils.

\section{Study period}

This study was conducted for 38 days from June 8 to July 15, 2015 in the order of pretest, oil blending and maturing, aroma experiment, and posttest. The experimental group wore the necklaces for 2 weeks from June 29 to July 10,

\section{Evaluation tool}

The pretest was conducted to determine the general characteristics of the subjects, and their job stress, depression and fatigue were examined before and after the experiment to determine to effect of essential oil. All evaluations were done by the subjects through self-testing.

\section{(1) Job stress}

The tool developed by Chang et al. (2005) in Korea Occupational Safety \& Health Agency was used in this study. This tool consists of total 43 items in 8 categories about job stress, such as physical environment, job demand, job autonomy, relationship conflict, job insecurity, organizational management system, inappropriate compensation, and workplace culture. Each item is rated on a four-point scale (4 points for highest stress and 1 point for lowest stress), with higher scores indicating relatively higher stress. The reliability was Cronbach's $\alpha=.785$.

\section{(2) Depression}

The Beck Depression Inventory (BDI) by Beck et al. (1961) is a self-report questionnaire consisting of total 21 items.

Table 1. Main ingredients and blending ratio of oils for this study.

\begin{tabular}{|c|c|c|c|c|c|c|}
\hline Aroma oil & Sort & Origin & Extracted parts & Extraction method & Main chemistry ingredients & Ratio \\
\hline Bergamot & Top note & Italy & Peel of fruits & Expression & $\begin{array}{c}\text { Linalyl acetate } 32 \% \text {, } \\
\text { Limonene } 33 \% \text {, } \\
\text { Linalool } 14 \% \text {, } \\
\text { Bergaptene } 0.23 \%\end{array}$ & 3 \\
\hline Lavender & Middle note & $\begin{array}{c}\text { Shore of the } \\
\text { Mediterranean }\end{array}$ & $\begin{array}{l}\text { Surrounding } \\
\text { buds }\end{array}$ & $\begin{array}{c}\text { Steam } \\
\text { distillation }\end{array}$ & $\begin{array}{c}\text { Linalyl acetate } 46.71 \sim 53.80 \% \\
\text { Linalool } 29.3 \sim 41.62 \% \\
\text { Camphor } 0.3 \%\end{array}$ & 2 \\
\hline Sandalwood & Base note & India & Trunk, root & Steam distillation & $\begin{array}{c}\text { Santalol } 70 \sim 90 \% \text {, } \\
\text { Borneol } 5 \%\end{array}$ & 1 \\
\hline
\end{tabular}


This study used the Korean version of the BDI adapted by Lee et al. (1995). The BDI comprehensively measures cognitive, emotional, motivational and physical symptoms as well as decreased self-esteem. Each item is rated on a scale of $0-3$, which can be distributed from 0 to 63 . Higher scores indicate greater depression. The reliability was Cronbach's $\alpha$ $=.906$.

\section{(3) Fatigue}

Fatigue was measured with the Multidimensional Fatigue Scale (MFS) restructured by Chang (2000) with sub-factors such as overall fatigue level, daily life dysfunction, situational fatigue, sleep, and sensitivity to relaxation, based on the FAI (Fatigue Assessment Inventory) developed by Schwartz et al. (1993) with verified reliability and validity. MFS consists of total 19 items such as 8 items in overall fatigue including "I lack efficiency in time-consuming tasks when exhausted," 6 items in daily life dysfunction including "I feel drowsy and sleepy when exhausted," and 5 items in situational fatigue including "I feel exhausted when stressed." They were rated on a seven-point scale (strongly disagree 1-2-3-4-5-6-7 strongly agree), and the greater the sum of scores in each factor, the higher the level of fatigue. The reliability was Cronbach's $\alpha=.923$.

\section{Data analysis}

Collected data were statistically analyzed using SPSS WIN 18.0, and the homogeneity between the two groups (control and experimental) was analyzed by an independent t-test. Pretest-posttest changes in job stress, depression and fatigue within the groups were analyzed by a paired t-test.

\section{Research procedures}

This study was conducted in the order of pre-test, experiment and post-test after the pre-survey to determine the effect of essential oil extracted from herbs on job stress, depression and fatigue of hospital workers. To conduct this study, the researchers received education and training in aroma instructor course about the objective of this study, data collection method and how to inhale aroma from necklaces from May 12 to June 17, 2015, and also received the Aroma Instructor Course Level 2 license from the Korea Aromatherapy Instructor Association. Essential oils to relieve job stress, depression and fatigue were selected based on literature review (Kwan et al., 2008; Sakong and Kim, 2006). Aroma-related research data were analyzed and advice was sought from 1 aroma therapy expert and 1 principal researcher at a local community herb research center to blend the top, middle and base note into the ratio of 3:2:1. To determine whether the aroma necklace inhaling method is suitable for hospital workers, a preliminary test was held on 4 workers who inhaled the necklaces according to the method and recorded inconveniences for modification and improvement.

The pretest first surveyed the general characteristics using a questionnaire in the experimental group that suits the objective of this study, after which job stress, depression and fatigue were measured. The same was done for the control group. The control group and experimental group were randomly formed and measured with the same ratio of departments among the ordinary workers with the same working patterns to secure reliability of the effect. First, the researchers explained the objective and content of the study to the subjects before inhaling the aroma, and the subjects filled out the questionnaire after providing written consent. The questionnaire was explained further when necessary.

Aroma is not accumulated in the body but is discharged through respiratory organs, liver and kidneys, but using the same aroma for 3 weeks to 3 months may accumulate toxicity on liver or kidneys, and thus it is necessary to take a break for at least a week or use different aromas (Oh, 2002). Therefore, this study was conducted for 2 weeks according to the 
expert advice and the schedule of the experimental group, and the subjects were to wear the necklaces only at work so that the experimental environment can be controlled. Moreover, the researchers distributed the guidelines for the experiment to the experimental group so that the subjects do not forget to wear the necklaces, and also provided a text message notification service every day to wear the necklaces.

In the posttest, the researchers measured stress, depression and fatigue with a questionnaire for both groups in the same method, and added a survey on experiment satisfaction for the experimental group. For the control group, aroma necklaces with the same blend of aroma essential oil used on the experimental group were provided as a gift after the study for ethical fairness and gratitude, along with training about how to use them. Fig. 1 shows the detailed activities in each procedure.

\section{Results and Discussions}

\section{General characteristics of subjects}

To determine the effect of essential oil extracted from herbs on job stress, depression and fatigue of hospital workers,
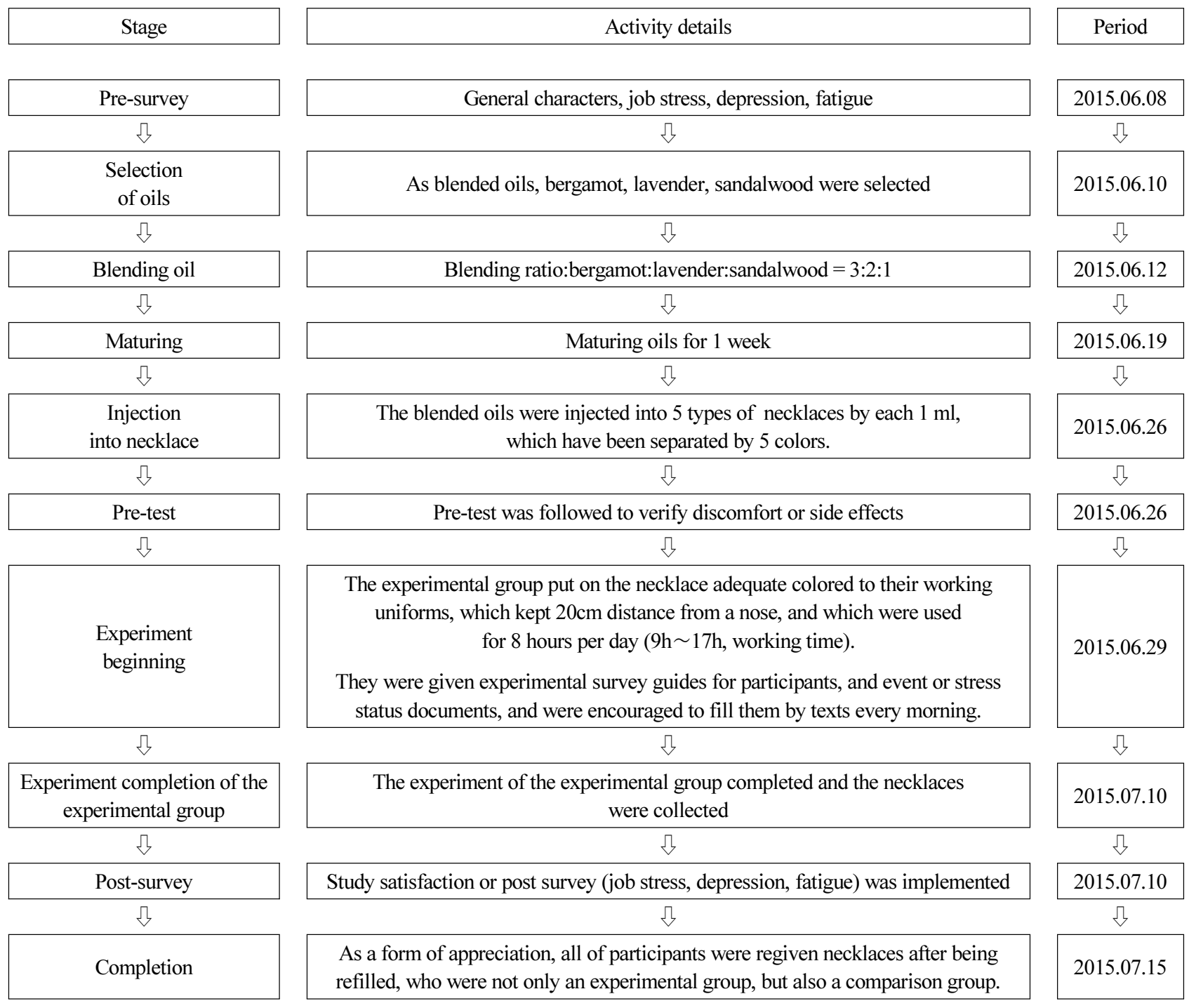

Figure 1. Detailed activity by research procedures. 
Table 2. General characteristics of study subjects.

\begin{tabular}{|c|c|c|c|c|}
\hline \multirow{2}{*}{ Characteristics } & \multirow{2}{*}{ Sort } & Total & Cont. $(n=30)$ & Exp. $(n=30)$ \\
\hline & & $\mathrm{n}(\%)$ & $\mathrm{n}(\%)$ & $\mathrm{n}(\%)$ \\
\hline \multirow{4}{*}{ Age } & $20 \mathrm{~s}$ & $4(6.7)$ & $4(6.7)$ & $0(0)$ \\
\hline & $30 \mathrm{~s}$ & $16(26.7)$ & $11(18.3)$ & $5(8.3)$ \\
\hline & 40s & $26(43.3)$ & $10(16.7)$ & $16(26.7)$ \\
\hline & $50 \mathrm{~s}$ & $14(23.3)$ & $5(8.3)$ & $9(15)$ \\
\hline \multirow{2}{*}{ Gender } & Male & $8(13.3)$ & $4(6.7)$ & $4(6.7)$ \\
\hline & Female & $52(86.7)$ & $26(43.3)$ & $26(43.3)$ \\
\hline \multirow{4}{*}{$\begin{array}{l}\text { Final academic } \\
\text { background }\end{array}$} & Highschool & $4(6.7)$ & $3(5)$ & $1(1.7)$ \\
\hline & College & $35(58.3)$ & $19(31.7)$ & $16(26.7)$ \\
\hline & University for 4 years & $13(21.7)$ & $5(8.3)$ & $8(13.3)$ \\
\hline & More than Master degree & $8(13.3)$ & $3(5)$ & $5(8.3)$ \\
\hline \multirow{2}{*}{ Marriage status } & Married & $50(83.3)$ & $23(38.3)$ & $27(45)$ \\
\hline & Unmarried & $10(16.7)$ & $7(11.7)$ & $3(5)$ \\
\hline \multirow{2}{*}{ Religion } & Yes & $34(56.7)$ & $13(21.7)$ & $21(35)$ \\
\hline & No & $26(43.3)$ & $17(28.3)$ & $9(15)$ \\
\hline \multirow{2}{*}{ Monthly earning } & under 300 & $27(45)$ & $20(33.3)$ & $7(11.7)$ \\
\hline & more than 300 & $33(55)$ & $10(16.7)$ & $23(38.3)$ \\
\hline \multirow{2}{*}{ Smoking } & Yes & $2(3.3)$ & $2(3.3)$ & $0(0)$ \\
\hline & No & $58(96.7)$ & $28(46.7)$ & $30(50)$ \\
\hline \multirow{2}{*}{ Coffee intake } & Yes & $8(13.3)$ & $5(8.3)$ & $3(5)$ \\
\hline & No & $52(86.7)$ & $25(41.7)$ & $27(45)$ \\
\hline \multirow{2}{*}{ Drinking } & Yes & $35(58.3)$ & $18(30)$ & $17(28.3)$ \\
\hline & No & $25(41.7)$ & $12(20)$ & $13(21.7)$ \\
\hline \multirow{5}{*}{ Healthy status } & Very healthy & $5(8.3)$ & $5(8.3)$ & $0(0)$ \\
\hline & Healthy & $26(43.3)$ & $13(21.7)$ & $13(21.7)$ \\
\hline & Normal & $27(45)$ & $11(18.3)$ & $16(26.7)$ \\
\hline & Not healthy & $1(1.7)$ & $1(1.7)$ & $0(0)$ \\
\hline & Not healthy too much & $1(1.7)$ & $0(0)$ & $1(1.7)$ \\
\hline \multirow{2}{*}{ Taking medicines } & Yes & $16(26.7)$ & $9(15)$ & $7(1.7)$ \\
\hline & No & $44(73.3)$ & $21(35)$ & $23(38.3)$ \\
\hline \multirow{4}{*}{ Menstruation status } & Regularly & $32(53.3)$ & $19(31.7)$ & $13(21.7)$ \\
\hline & Irregularly & $8(13.3)$ & $2(3.3)$ & $6(10)$ \\
\hline & Menopause & $12(20)$ & $5(8.3)$ & $7(11.7)$ \\
\hline & Male & $8(13.3)$ & $4(6.7)$ & $4(6.7)$ \\
\hline \multirow{2}{*}{ Losing weight } & Yes & $6(10)$ & $3(5)$ & $3(5)$ \\
\hline & No & $54(90)$ & $27(45)$ & $27(45)$ \\
\hline \multirow{3}{*}{ Life satisfaction } & Satisfied & $33(55)$ & $19(31.7)$ & $14(23.3)$ \\
\hline & Usual & $24(40)$ & $10(16.7)$ & $14(23.3)$ \\
\hline & Unsatisfied & $3(5)$ & $1(1.7)$ & $2(3.3)$ \\
\hline \multirow{3}{*}{ Occupation type } & Medical technicians & $15(25)$ & $6(10)$ & $9(15)$ \\
\hline & Nurses & $38(63.3)$ & $17(28.3)$ & $21(35)$ \\
\hline & General administrations & $7(11.7)$ & $7(11.7)$ & $0(0)$ \\
\hline
\end{tabular}


this study examined total 60 subjects, 30 each for the control group and experimental group. Table 2 shows the general characteristics of the subjects. By gender, $13.3 \%$ were men and $86.7 \%$ were women. $6.7 \%$ were in their $20 \mathrm{~s}, 26.7 \%$ in their $30 \mathrm{~s}, 43.3 \%$ in their $40 \mathrm{~s}$, and $23.3 \%$ in their $50 \mathrm{~s}$. This indicates that there were 6 times more women than men, and $66.6 \%$ were in their $40 \mathrm{~s}$ or older. Most of them were college graduates $(58.3 \%)$, followed by 4 -year university graduates (21.7\%), masters or higher (13.3\%), and high school graduates (6.7\%). 83.3\% were married, and 56.7\% had a religion. $45 \%$ had a monthly income lower than 3 million KRW and 55\% had 3 million KRW or higher. 3.3\% were smokers, $13.3 \%$ had coffee intake, and $58.3 \%$ was drinking. As for satisfaction in life, $55 \%$ were satisfied, $40 \%$ were neutral, and $5 \%$ were unsatisfied. By occupation, $25 \%$ were medical technicians, $63.3 \%$ were nurses, and $11.7 \%$ were in general administration.

\section{Between-group test for homogeneity}

To determine the effect of wearing aroma necklaces with essential oil extracted from herbs on job stress, depression and fatigue of hospital workers, an experiment was conducted in two groups, control and experimental. Table 3 shows the result of data analysis with an independent t-test on job stress, depression and fatigue to determine the homogeneity of the two groups.

In the case of job stress, the control group had an average score of $101.2 \pm 10.3$ and the experimental group had 104.9 \pm 9.9 , showing no statistically significant difference $(\mathrm{p}=.151)$. For depression, the control group had $29.4 \pm 9.0$ and the experimental group had $28.5 \pm 5.6$, showing no statistically significant difference $(\mathrm{p}=.657)$. For fatigue, the control group had $72.0 \pm 18.9$ and the experimental group had $70.5 \pm 19.0$, showing no statistically significant difference ( $\mathrm{p}=.771)$.

As a result, there was no statistically significant difference in all items of job stress, depression and fatigue between the two groups before the experiment, thereby confirming that the two groups are homogeneous.

\section{Within-group pre-post test changes}

To determine the effect of wearing aroma necklaces with essential oil extracted from herbs on job stress, depression and fatigue of hospital workers, this study conducted a measurement of the two groups once each before and after the experiment, based on which the changes in job stress, depression and fatigue are examined.

\section{Changes in job stress}

Table 4 shows the result of analyzing the changes in job stress for the two groups with a paired t-test. For the control group, job stress had no statistically significant difference from average 101.2 \pm 10.3 to $104.1 \pm 11.3$ ( $p=0.086$ ), and the experimental group wearing the aroma necklaces with essential oil extracted from herbs also had no statistically

Table 3. Pre-equivalence study between the comparison group and the experimental group.

\begin{tabular}{lcccc}
\hline Item & $\begin{array}{c}\text { Cont. }(\mathrm{n}=30) \\
(\text { score })\end{array}$ & $\begin{array}{c}\text { Exp. }(\mathrm{n}=30) \\
(\text { score })\end{array}$ & $\mathrm{t}$ & $p$ \\
\hline Job stress & $101.2 \pm 10.3^{\mathrm{z}}$ & $104.9 \pm 9.9$ & -1.454 & $.151^{\mathrm{NS}}$ \\
Depression & $29.4 \pm 9.0$ & $28.5 \pm 5.6$ & .447 & $.657^{\mathrm{NS}}$ \\
Fatigue & $72.0 \pm 18.9$ & $70.5 \pm 19.0$ & .293 & $.771^{\mathrm{NS}}$ \\
\hline
\end{tabular}

${ }^{\mathrm{z}}$ Mean \pm standard deviation.

${ }^{\mathrm{NS}}$ Non signigicant at $p<.05$ by independent t-test. 
Table 4. Statistic analysis of job stress changes of hospital workers after inhaling essential oils by wearing necklaces.

\begin{tabular}{lcccc}
\hline Group & Pre-test (score) & Post-test (score) & t & $p$ \\
\hline Cont. $(\mathrm{n}=30)$ & $101.2 \pm 10.3^{\mathrm{z}}$ & $104.1 \pm 11.3$ & -1.779 & $0.086^{\mathrm{NS}}$ \\
Exp. $(\mathrm{n}=30)$ & $104.9 \pm 9.0$ & $104.7 \pm 9.8$ & 0.185 & $0.855^{\mathrm{NS}}$ \\
\hline
\end{tabular}

${ }^{\mathrm{z}}$ Mean \pm standard deviation.

${ }^{\mathrm{NS}}$ Non signigicant at $p<.05$ by paired t-test.

significant difference from average $104.9 \pm 9.0$ before experiment to $104.7 \pm 9.8$ after $(p=0.855)$. This is similar to the study by Oh (2012) on female production workers working in shifts and the study by Choi and Lee (2012) on patients with essential hypertension, both of which claimed that there was no statistical difference between the two groups after aroma experiment. However, studies by Sung (2005) and Lee (2012) on nurses in operating rooms applying aroma inhalation claimed that there was a positive change in stress, and the study by Jang (2009) on nurses in emergency rooms also argued that there was a significant difference between the two groups and aroma was effective on reducing job stress.

This study failed to obtain such effect on job stress because all hospitals were in emergency due to MERS-CoV during the study period, and the relevant hospital was designated as an intensive medical center for the disease, while at the same time it was also the medical audit period. Furthermore, there were 34 days of labor-management strike and conflicts against the reckless management of public institutions of national university hospitals. These circumstances constantly increased stress factors, but this study limited the experiment only to working hours to inhale the aroma, which may have limited the effect on reducing job stress.

\section{Changes in depression}

Table 5 shows the result of analyzing the changes in depression for the two groups with a paired t-test. For the control group, depression had no statistically significant difference from average $29.4 \pm 9.0$ to $28.8 \pm 8.0$ ( $p=0.486$ ), but the experimental group wearing the aroma necklaces with essential oil extracted from herbs showed a statistically significant $(\mathrm{p}=0.017)$ decrease of depression from average $28.5 \pm 5.6$ to $26.63 \pm 5.3$ after the experiment. This is similar the study by Lee (2012) on nurses in the operating rooms applying aroma inhalation, proving that it reduced stress, fatigue and depression of the nurses and enabled them to work in a more relaxing state. It is also consistent with the study by Oh (2012) on female production workers working in shifts, where aroma roll-on inhalation showed a significant effect on decreasing depression and improving sleep state. The oils used in this study are bergamot to lift up the mood and relieve depressed feelings and lavender to soothe and relax the mind, which are both effective for stress, anxiety, insomnia and headaches (Sakong and Kim, 2006). As such, essential oils extracted from herbs are used as a therapy to relax and vitalize the body and soul of the subjects.

Table 5. Statistic analysis of depression changes of hospital workers after inhaling essential oils by wearing necklaces.

\begin{tabular}{lcccc}
\hline Group & Pre-test (score) & Post-test (score) & t & $p$ \\
\hline Cont. $(\mathrm{n}=30)$ & $29.4 \pm 9.0^{\mathrm{z}}$ & $28.8 \pm 8.0$ & 0.706 & $0.486^{\mathrm{NS}}$ \\
Exp. $(\mathrm{n}=30)$ & $28.5 \pm 5.6$ & $26.6 \pm 5.3$ & 2.530 & $0.017^{*}$ \\
\hline
\end{tabular}

${ }^{\mathrm{z}}$ Mean \pm standard deviation.

${ }^{\mathrm{NS}}$ Non signigicant or significant $* p<0.05$ by paired t-test. 


\section{Changes in fatigue}

Table 6 shows the result of analyzing the changes in fatigue for the two groups with a paired t-test. For the control group, fatigue had no statistically significant difference from average $72.0 \pm 18.9$ to $75.7 \pm 19.7(p=0.156)$, but the experimental group wearing the aroma necklaces with essential oil extracted from herbs showed a statistically significant $(p=0.049)$ decrease of fatigue from average $70.5 \pm 19.0$ to $64.3 \pm 20.0$ after the experiment. This is consistent with the study claiming that aroma therapy reduces fatigue in nurses working in operating rooms and allows them to work in a more relaxed state, and that it can be an alternative therapy to increase work efficiency (Lee, 2012). It is also consistent with the study on nursing students in their first clinical training, which claimed that fatigue decreased after inhaling aroma through necklaces (Kang and Kim, 2002). The main ingredient of lavender and bergamot used in this study is ester (Linalyl acetate), which soothes and comforts the mind, thereby relaxing inner muscles. The balm used in aroma therapy promotes metabolism and naturally treats stress and diseases without side effects (Sakong and Kim, 2006).

This study is conducted to examine the effect of essential oil on job stress, depression and fatigue of hospital workers after wearing necklaces with essential oil extracted from herbs for 2 weeks. It was designed so that the subjects are to wear aroma necklaces that are easy to carry at work, so that they can immediately inhale the aroma in case of stress, which is an improved method suggested in previous research $(\mathrm{Oh}, 2012)$. The hospital workers put the necklaces on immediately after they got to work and kept them on until they got off work, but this failed to maintain the continuity of the experiment due to the five-day workweek system. Moreover, since the study was conducted without controlling external factors such as MERS-CoV and labor-management strike, there was difficulty in objectifying the level of job stress, depression and fatigue of hospital workers. Therefore, this study has limitations in generalizing the results and verifying that the same effect will be produced after conducting the same experiment on workers of other hospitals.

It is necessary to conduct research on methods to reduce job stress, depression and fatigue of hospital workers and on various programs to improve the quality of their lives. The inhalation method by wearing aroma necklaces also requires some improvement. A replication study must be conducted so that the subjects can select the aroma of their choice in light of their preferences for fragrances, and follow-up research must be designed to have the subjects wear the aroma necklaces everywhere except for sleeping hours, instead of limiting only to working hours. Furthermore, the experiment should be conducted for at least 3-4 weeks, and the same shape of tool must be used because the size of the hole in the pendant may give off different intensity of aroma.

This study applied aroma therapy by controlling the subjects to ordinary hospital workers, but it can be improved by expanding the scope of subjects to shift workers, clinical nurses and workers in special departments. Different application methods and research designs can also be used to verify the effect. More systematic and diverse studies are anticipated with regard to aroma therapy including changes in chemical numbers of essential oil that can objectively verify the inhalation of aroma necklaces.

Table 6. Statistic analysis of fatigue degree changes of hospital workers after inhaling essential oils by wearing necklaces.

\begin{tabular}{lcccc}
\hline Group & Pre-test (score) & Post-test (score) & $\mathrm{t}$ & $p$ \\
\hline Cont. $(\mathrm{n}=30)$ & $72.0 \pm 18.9^{\mathrm{z}}$ & $75.7 \pm 19.7$ & -1.455 & $0.156^{\mathrm{NS}}$ \\
Exp. $(\mathrm{n}=30)$ & $70.5 \pm 19.0$ & $64.3 \pm 20.0$ & 2.055 & $0.049^{*}$ \\
\hline
\end{tabular}

${ }^{\mathrm{z}}$ Mean \pm standard deviation.

${ }^{\mathrm{NS}}$ Non signigicant or significant $* p<0.05$ by paired t-test. 


\section{Conclusion}

To determine the effect of wearing aroma necklaces with essential oil extracted from herbs as an alternative therapy on job stress, depression and fatigue of hospital workers, this study selected total 60 subjects -30 each for the control group and experimental group - as the final subjects. The subjects are ordinary workers at K University Hospital in D city that agreed to participate in this study who meet the study requirements. The experimental group wore necklaces with the pendants containing essential oil extracted from herbs hanging at a $20 \mathrm{~cm}$ distance from the nose during working hours $(8$ hours a day) for 2 weeks from June 29 to July 10, 2015. After the experiment, job stress changed from $101.2 \pm 10.3$ to $104.1 \pm 11.3$ in the control group, and $104.9 \pm 9.0$ to $104.7 \pm 9.8$ in the experimental group, showing no statistically significant difference in both groups. Depression did not show a significant change in the control group from $29.4 \pm 9.0$ to 28.8 \pm 8.0 , whereas it decreased significantly from $28.5 \pm 5.6$ to $70.5 \pm 19.0$ in the experimental group $(p=.017)$. Fatigue had no significance in the control group from $72.0 \pm 19.0$ to $75.7 \pm 19.7$, whereas it decreased significantly from $70.5 \pm 19.0$ to $64.3 \pm 20.0$ in the experimental group ( $p=.049)$. This shows that essential oil extracted from herbs is effective in reducing depression and fatigue of ordinary workers at hospitals. However, even though the study was designed to immediately inhale the aroma in cases of stress by carrying it around at work, the continuity of experiment could not be maintained due to the five-day workweek system as well as environmental factors (internal and external) in the hospital during the research. Therefore, there is a need for more systematic research on aroma therapy that is recently receiving spotlight as an alternative therapy by solving these problems.

\section{References}

Beck, A.T., C.H. Ward, M. Mendelson, J. Mock, and J. Erbaugh. 1961. An inventory for measuring depression. Archives of General Psychiatry 4:561-571.

Chang, S.J. 2000. Standardization of health data collection measurement. Gyechuk munhwasa, Seoul, Korea.

Chang, S.J. S.B. Koh, M.G. Kang, B.S. Cha, J.K. Park, S.J. Hyun, J.H. Park, S.A. Kim, D.M. Kang, S.S. Chang, K.J. Lee, E.H. Ha, J.M. Woo, J.J. Cho, H.S. Kim, and J.S. Park. 2005. Epidemiology of psychosocial distress in Korean employees. Journal Preventive Medicine Public Health 38(1):25-37.

Choi, E.M. and K.S. Lee. 2012. Effects of aroma inhalation on blood pressure, pulse rate, sleep, stress, and anxiety in patients with essential hypertension. Journal of Korean Biological Nursing Science 14(1):41-48.

Jang, E.H. 2009. The effect of aromatic inhalation therapy to emergency care-unit nurse stress and fatigue focusing on the dry inhalation therapy. MS thesis, Gachon University of Medicine and Science, Incheon, Korea.

Kang, J.Y. and K.S. Kim. 2002. Effect of aromatherapy on anxiety and fatigue in students nurses experiencing their first clinical practice. Journal of the Korean Academy of Fundamentals of Nursing 9(2):226-236.

Kim, M.H., J.I. Kim, and E. Ha. 2014. Effects of aroma-necklace application on perceived stress, symptoms of stress and changes in autonomic nervous system among nursing students in clinical training. Journal of Korean Biological Nursing Science 16(4):334-341.

Kim, Y.H. and K.H. Suh. 2011. Relationships between job stresses, ego-resilience, stress responses, and quality of service among hospital employees. Korean J. Health Psychol. 16(2):329-345.

Kwan, S.Y., S.Y. Kim, H.J. Kim, J.H. Kim, and K.M. Yu. 2008. The complete guide to aromatherapy. Hynmonsa, Seoul, Korea.

Lee, M.K., Y.H. Lee, S.H. Park, C.H. Song, Y.J. Jeong, S.K. Hong, B.K. Lee, P.L. Jang, and E.L. Yun. 1995. Reliability and factor analysis-A standardization study of beck depression inventory 1-Korean version (K-BDI). The Korean Journal of Psychopathology 4(1):77-95. 
Lee, S.M. 2012. The effects of aromatherapy on sress, fatigue, and depression of operating room nurses. MS thesis, Eulji University, Daejeon, Korea.

Oh, H.G. 2002. Aromatherapy hand book-A perfect guide for professionals. Yangmun, Seoul, Korea.

Oh, Y.M. 2012. The effects of aroma inhalation method with roll-on in occupation stress, depression and sleep in female manufactures workers. MS thesis, Seonam University, Namwon, Korea.

Sakong, J.K. and Y.H. Kim. 2006. Aromatherapy. Hyunmoonsa, Seoul, Korea.

Schwartz, J.E., L. Jandorf, and L.B. Krupp. 1993. The measurement of fatigue: A new instrument. J. Psychosom. Res. 37(7):753-762.

Statistics Korea. 2014. Report on the social survey (Health • education·safety•family•environment). Daejeon, Korea: Statistics Korea.

Sung, S.N. 2005. The effects of aroma inhalation method on stress of nurses working in operating room. MS thesis, Gyeong-Sang National University, Jinju, Korea.

Yu, K.Y. 2010. The association between job stress and psychosocial stress in young male and female manufacture workers. MS thesis, Ajou University, Suwon, Korea. 